\title{
Candida glabrata infection of urinary bladder in a Chinchilla Persian cat
}

\author{
Seungji Woo, Hak-Hyun Kim, Ji-Houn Kang*, Ki-Jeong Na, Mhan-Pyo Yang \\ College of Veterinary Medicine and Veterinary Medical Center, Chungbuk National University, Cheongju 28644, Korea
}

(Received: February 10, 2017; Revised: April 22, 2017; Accepted: May 11, 2017)

\begin{abstract}
A 5-year-old castrated male Chinchilla Persian cat weighing $4.84 \mathrm{~kg}$ was referred for hematuria. The cat had a history of urethrostomy and bacterial cystitis. In urine culture, Candida glabrata was cultured on Sabouraud dextrose agar. Based on these results, the cat was diagnosed with Candida cystitis. Subsequently, oral administration of fluconazole was initiated. Urine culture was negative at 31 days after administration. This case describes the diagnosis and treatment of Candida glabrata infection of urinary bladder in a cat with a history of urethrostomy.
\end{abstract}

Keywords: Candida glabrata, cat, hematuria, urinary bladder

Candida spp. is composed of more than 150 species of budding yeasts [3]. Candida spp. is ubiquitously found on many plants and normal flora of dogs and cats [7]. However, pathogenic Candida spp. infections can occur under immune compromised condition [6].

The most common disease-causing Candida spp. in dogs and cats is Candida (C.) albicans. However, several reports have demonstrated an increase of non- $C$. albicans species isolated from both healthy and ill animals [1, 6, 10, 13]. In cats, Candida spp. infection most commonly results in cutaneous or lower urinary tract infection. Gastrointestinal, ocular, and systemic infections occur less frequently [3].

The term feline lower urinary tract disease (FLUTD) is used to describe the group of clinical signs related to problems in voiding urine [8]. Clinical signs include hematuria, pollakiuria, stranguria, periuria, and urethral obstruction [5]. The most common cause of FLUTD is feline idiopathic cystitis (FIC). A study reported that $8 \%$ of 77 cats were due to bacterial infection [5], suggesting infection is relatively uncommon in cats with LUTD. Less than $1 \%$ of urinary tract infections in cats are due to Candida spp. infections [11]. $C$. glabrata is an extremely rare causative agent. Although there are a few case reports of $C$. glabrata infection of urinary tract in cats, no report has described infection in cats under normal immune status. Herein, we describe the diagnosis and treatment of $C$. glabrata infection of urinary bladder in a cat having a history of urethrostomy due to obstructive FLUTD.

A 5-year-old castrated male Chinchilla Persian cat weighing $4.84 \mathrm{~kg}$ was presented with hematuria. The patient was referred for intermittent hematuria with a history of urethrostomy and bacterial cystitis. At presentation, physical exami-

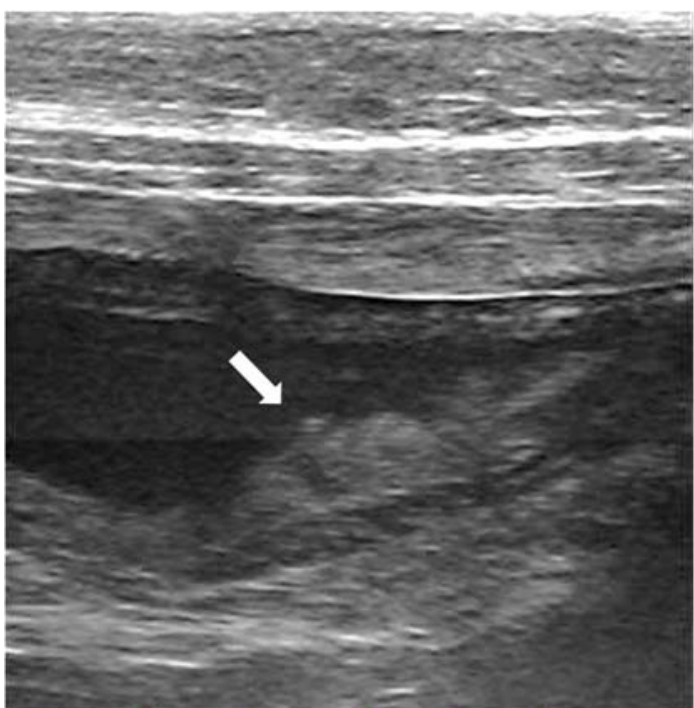

Fig. 1. An ultrasonographic image obtained from a Chinchilla Persian cat with Candida cystitis. Thickening and irregular margin of urinary bladder are observed. Mobile blood clots (arrow) within urinary bladder are suspected.

nation and results of blood analyses were non-remarkable. Abdominal ultrasonography revealed thickening and irregular margin of urinary bladder wall, and sediments showing hyperechoic and non-shadowing echogenicity of bladder lumen were suspected to mobile blood clots (Fig. 1). Urine was reddish brown in color. Urine specific gravity (1.049) was normal. There was no causative agent on microscopic examination of urine. Based on the previous history of bacte-

\footnotetext{
*Corresponding author

Tel: +82-43-261-3691, Fax: +82-43-269-2595

E-mail: jhkang@chungbuk.ac.kr
} 


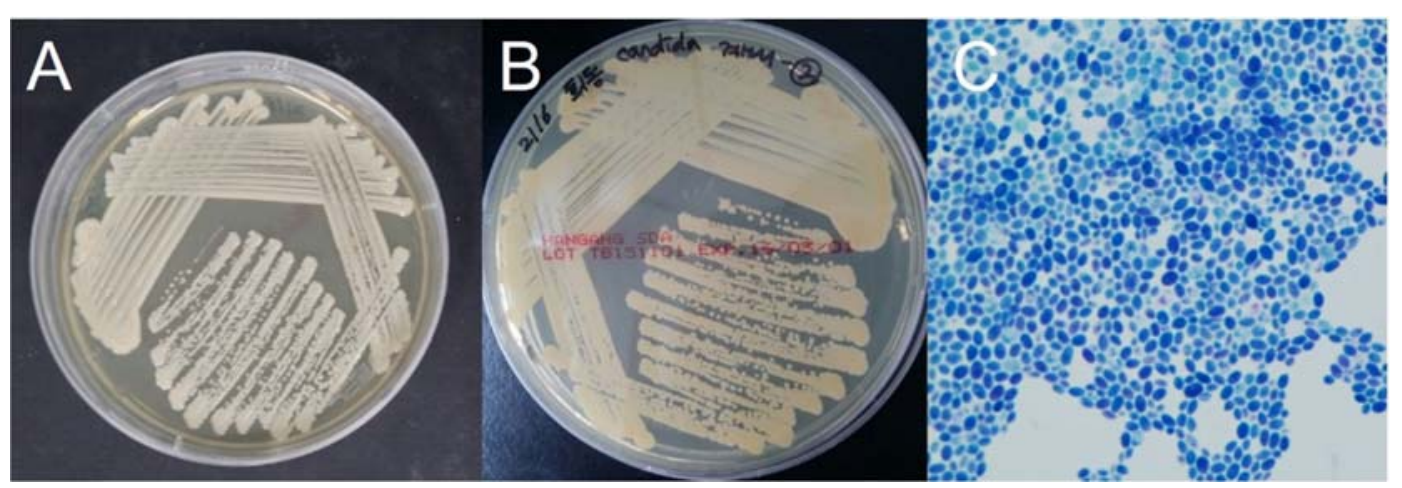

Fig. 2. Culture of Candida (C.) glabrata on Sabouraud dextrose agar (A and B). C. glabrata is observed with characterization of cream color and small round blastoconidia. Microscopically, C. glabrata is observed using Diff-Quik stain (C).

rial cystitis, the cat was administered with antibiotic drug, amoxicillin-clavulanic acid (Amocla tab, $25 \mathrm{mg} / \mathrm{kg}$, per orally [PO], twice a day [bid]; Kuhnil Pharmaceutical, Korea) and $\mathrm{H}_{2}$ receptor antagonist, ranitidine (Ranitidine $\mathrm{HCl}$ tab, 3.5 $\mathrm{mg} / \mathrm{kg}$, PO, bid; Wooridul Pharmaceutical, Korea) for 3 days. However, 3 days after initiating the administration, clinical sign of hematuria was not resolved, and the urine culture at presentation was negative for bacterial growth, but fungal infection was identified. A definitive diagnosis of fungal infection was made by identifying organisms in urine sample. The urine sample was centrifuged at $400 \times \mathrm{g}$ for $20 \mathrm{~min}$ and sediment was inoculated on sabouraud dextrose agar. The agar plates were incubated at $37^{\circ} \mathrm{C}$ under aerobic condition in $5 \%$ to $7 \%$ carbon dioxide for 2 weeks. Fungi were identified with a commercial test kit and stain. C. glabrata was identified by using an API 20C AUX commercial kit (bioMérieux, France). Based on results of this kit, biochemical reactions indicating $C$. glabrata infection were found, including fermentation and assimilation of glucose and trehalose [6]. From fungal culture, growth of C. glabrata was macroscopically observed with characteristics of cream color and small round blastoconidia (1 to $4 \mathrm{~mm}$ ) (Fig. 2A and B). Microscopically, budding and no-hyphae formation were observed using Diff-Quik stain (Fig. 2C). With a diagnosis of C. glabrata infection, antibacterial drug was replaced with antifungal drug, fluconazole (Furacan, $5 \mathrm{mg} / \mathrm{kg}$, PO, bid; Myungmoon Pharm, Korea).

Administration of $\mathrm{H}_{2}$ receptor antagonist was maintained. All these drugs were prescribed for 30 days. At 31th day after the administration, re-examination revealed no clinical sign. Repeated urine culture result was negative for fungal growth.

This report describes a case of $C$. glabrata infection of urinary bladder in a Chinchilla Persian cat having a history of urethrostomy. According to previous studies, urinary tract infections caused by Candida spp. account for less than $1 \%$ of all urinary tract diseases in cats [11]. C. albicans is the most common infectious agent causing Candida spp. urinary tract infection. However, infection caused by C. glabrata is very rare. In this case, we considered several possibilities causing this infection including weakened urothelial barrier, use of silicone or latex material and urethrostomy.

Cats with candida urinary tract infection including C. glabrata show typical clinical signs of lower urinary tract infection, with hematuria in $50 \%$ of cases $[3,9]$. Clinical signs of hematuria might be due to injury of bladder mucosa. In cats and humans, normal bladder layers consist of glycosaminoglycan (GAG) layer, urothelium, and muscular layer [8]. The term urothelium has been used to describe the epithelia covering the mucosal surface of most urinary tract [5]. GAG layer components and cell-to-cell adhesion molecules in the urothelium may function as a barrier [7]. This barrier might have been weakened by previous bacterial cystitis, subsequently allowing the invasion of $C$. glabrata.

In addition to defected barrier function, there are two widely cited potential virulence factors that contribute to the pathogenicity of $C$. glabrata [14]. The two factors are adhesion coded by epithelial adhesion (EPA) genes and microbial biofilms [14]. Adherence is critical to successful infection. It might be facilitated by exposing receptors to which Candida spp. can bind [4]. In the infectious process, the invasion of tissue commences when burrowing of hyphae begins through layers of cells [4]. However, C. glabrata does not develop hyphae except under extreme in vitro cultural condition. A major contributor of virulence and adherence to host cell is EPA1 gene [2]. Moreover, it has been reported that nicotinic acid deficiency is associated with adherence of C. glabrata [4]. Privation of nicotinic acid has been shown to signal the expression of a lectin encoded by the yeast's EPA1 gene, thus enhancing its adherence to bladder mucosal cell [10]. Because several articles such as cat toy and sand catcher are made of silicone or latex material, persistent existence of pathogen on silicon or latex with low nicotinic acid might play major roles in C. glabrata infection [4]. Furthermore, microbial biofilm may help facilitate fungal pathogen by evading host defense mechanisms, resisting fungal treatment, and withstanding competitive pressure from other organisms [15].

C. glabrata can enter the urinary tract by ascending from the perineum (retrograde infection) or by hematogenously seeding the kidney and "spilling over" into the urine (ante- 
grade infection) [4]. In the present case, C. glabrata infection might be retrograde infection because no systemic sign compatible with antegrade infection was revealed. Permanent urethral opening after perineal urethrostomy might have contributed to C. glabrata infection. However, there are few reports of urethrostomy as a predisposing factor in Candida spp. Infection $[9,13]$. Several factors associated with perineal urethrostomy may predispose to ascending bacterial urinary tract infection [12]. These factors include decreased length of urethra, loss of penile urethral mucosal defense mechanisms, increased diameter of external urethral orifice, impaired striated urethralis muscle function, and decreased intraluminal pressure [12]. However, these factors are limited to bacterial urinary tract infections. Further studies are needed to determine whether aforementioned factors caused by urethrostomy might influence fungal infection of urinary tract.

Clinicians often overlook the possibility of $C$. glabrata infection because the pathogen is very rarely observed. Nevertheless, C. glabrata may remain in silicon or latex material and become pathogenic. Retroinfection can occur in cats whose urinary bladder has been weakened by recurrent urinary tract infections. Therefore, $C$. glabrata infection should be considered as a differential diagnosis in patients with clinical symptoms indicating urinary tract infection.

\section{Acknowledgments}

This research was supported by the Basic Science Research Program through the National Research Foundation (NRF) of Korea, which was funded by the Ministry of Science, ICT and Future Planning (NRF-2016R1A1A1A05005395).

\section{References}

1. Bailiff NL, Nelson RW, Feldman EC, Westropp JL, Ling GV, Jang SS, Kass PH. Frequency and risk factors for urinary tract infection in cats with diabetes mellitus. J Vet Intern Med 2006, 4, 850-855.

2. Bailiff NL, Westropp JL, Nelson RW, Sykes JE, Owens SD, Kass PH. Evaluation of urine specific gravity and urine sediment as risk factors for urinary tract infections in cats. Vet Clin Pathol 2008, 37, 317-322.

3. Fidel PL Jr, Vazquez JA, Sobel JD. Candida glabrata: review of epidemiology, pathogenesis, and clinical disease with comparison to C. albicans. Clin Microbiol Rev 1999, 12, 80-96.

4. Fisher JF, Kavanagh K, Sobel JD, Kauffman CA, Newman CA. Candida urinary tract infection: pathogenesis. Clin Infect Dis 2011, 52 (Suppl 6), S437-451.

5. Gerber B, Boretti FS, Kley S, Laluha P, Müller C, Sieber $\mathbf{N}$, Unterer S, Wenger M, Flückiger M, Glaus T, Reusch CE. Evaluation of clinical signs and causes of lower urinary tract disease in European cats. J Small Anim Pract 2005, 46, 571-577.

6. Greene CE. Infectious Diseases of the Dog and Cat. 4th ed. pp. 666-672, Saunders, Philadelphia, 2012.

7. Hauser PJ, VanGordon SB, Seavey J, Sofinowski TM, Ramadan M, Abdullah S, Buffington CAT, Hurst RE. Abnormalities in expression of structural, barrier and differentiation related proteins, and chondroitin sulfate in feline and human interstitial cystitis. J Urol 2015, 194, 571-577.

8. Hostutler RA, Chew DJ, DiBartola SP. Recent concepts in feline lower urinary tract disease. Vet Clin North Am Small Anim Pract 2005, 35, 147-170.

9. Jin Y, Lin D. Fungal urinary tract infections in the dog and cat: a retrospective study (2001-2004). J Am Anim Hosp Assoc 2005, 41, 373-381.

10. Lagrotteria D, Rotstein C, Lee $\mathbf{C H}$. Treatment of candiduria with micafungin: a case series. Can J Infect Dis Med Microbiol 2007, 18, 149-150.

11. Ling GV, Norris CR, Franti CE, Eisele PH, Johnson DL, Ruby AL, Jang SS. Interrelations of organism prevalence, specimen collection method, and host age, sex, and breed among 8,354 canine urinary tract infections (1969-1995). J Vet Intern Med 2001, 15, 341-347.

12. Osborne CA, Caywood DD, Johnston GR, Polzin DJ, Lulich JP, Kruger JM, Ulrich LK. Feline perineal urethrostomy: a potential cause of feline lower urinary tract disease. Vet Clin North Am Small Anim Pract 1996, 26, 535-549.

13. Pressler BM, Vaden SL, Lane IF, Cowgill LD, Dye JA. Candida spp. urinary tract infections in 13 dogs and seven cats: predisposing factors, treatment, and outcome. $\mathrm{J}$ Am Anim Hosp Assoc 2003, 39, 263-270.

14. Rahimkhani M, Saberian M, Mordadi A, Varmazyar S, Tavakoli A. Urinary tract infection with Candida glabrata in a patient with spinal cord injury. Acta Med Iran 2015, 53, 516-517.

15. Rishpana MS, Kabbin JS. Candiduria in catheter associated urinary tract infection with special reference to biofilm production. J Clin Diagn Res 2015, 9, DC11-13. 